\title{
EDUCAÇÃO DIGITAL PARA ADULTOS EM PRIVAÇÃO DE LIBERDADE
}

\author{
José António Moreira ${ }^{1}$ \\ Universidade Aberta de Portugal \\ http://orcid.org/0000-0003-0147-0592
}

\section{RESUMO:}

$\mathrm{Na}$ atual sociedade do conhecimento e em rede, a educação, a capacitação tecnológica e as competências digitais são a chave para a plena integração do cidadão. Em Portugal, como na maioria dos países europeus, educação é um direito constitucional a ser garantido para todas as populações e em diferentes condições e espaços educativos, mesmo para os cidadãos que se encontram reclusos. A utilização de meios digitais é prevista e encorajada pelas diretrizes internacionais e pelas leis portuguesas, no entanto, a prática está longe de funcionar. Esta realidade evidencia a atualidade e pertinência deste texto e justifica a necessidade de produzir conhecimento acerca dos desafios educacionais e das potencialidades da Educação Digital enquanto estratégia para reduzir a discriminação, aumentar a justiça social, a inclusão e a qualificação em prisões nesta era digital. Neste estudo, apresentam-se práticas, experiências, programas e projetos de Educação Digital em prisões por todo o mundo, com destaque para o trabalho desenvolvido em Portugal pela Universidade Aberta (UAb) em parceria com a Direção Geral de Reinserção e Serviços Prisionais (DGRSP), relativo à criação e desenvolvimento do Campus Virtual Educonline@ pris, que se assume como uma academia de educação, formação, empregabilidade e cidadania digital. Pedagogicamente inspirado pelo modelo de aprendizagem da UAb, este Campus procura responder às necessidades educativas específicas dos estudantes em situação de privação de liberdade, respeitando as limitações legais inerentes à sua condição.

Palavras-chave: Educação digital. Prisões. Campus virtual.

\section{ABSTRACT:}

\section{DIGITAL EDUCATION FOR ADULTS IN DEPRIVATION OF FREEDOM}

In today's knowledge society education, technological empowerment and digital skills are the key to full citizen integration. In Portugal, as in most European countries, education is a constitutional right to be guaranteed for all populations and in different educational conditions and spaces, even for inmates. The use of digital media is provided for and encouraged by international guidelines and Portuguese law. However, the practice is far from working with many limitations. This reality highlights the relevance of the text we present and justifies the need to produce knowledge about the educational challenges and potential of Digital Education as a strategy to reduce discrimination, increase social justice, inclusion and qualification in prisons in this digital age. In this study, digital education programs and projects are presented in prisons around the world, highlighting the case of the work developed in Portugal by Universidade Aberta (UAb) in partnership with the General Directorate of Reinsertion and Prison Services (DGRSP), regarding the creation and development of the Educonline@Pris Virtual Campus, which is assumed as an academy of education, training, employability and digital citizenship. Pedagogically inspired by the UAb learning model, this Campus seeks to respond to the specific educational needs of students in situations of deprivation of liberty, respecting the legal limitations inherent to their condition.

Keywords: Digital education. Prisons. Inclusion. Virtual campus.

\footnotetext{
1 Doutor em Ciências da Educação (Universidade de Coimbra). Professor Associado do Departamento de Educação e Ensino a Distância (Universidade Aberta de Portugal). Investigador no Centro de Estudos Interdisciplinares do Século XX - CEIS20 (Universidade de Coimbra). E-mail: jmoreira@uab.pt
} 


\section{RESUMEN:}

\section{EDUCACIÓN DIGITAL PARA ADULTOS EN PRIVACIÓN DE LIBERTAD}

En la sociedad actual del conocimiento y las redes, la educación, la formación tecnológica y las competencias digitales son la clave para la plena integración del ciudadano. En Portugal, como en la mayoría de países europeos, la educación es un derecho constitucional que debe garantizarse para todas las poblaciones y en diferentes condiciones y espacios educativos, incluso para los ciudadanos que se encuentran presos. El uso de medios digitales está previsto y fomentado por las directrices internacionales y las leyes portuguesas, sin embargo, la práctica está lejos de funcionar. Esta realidad resalta la relevancia de este texto y justifica la necesidad de producir conocimiento sobre los desafíos educativos y el potencial de la Educación Digital como estrategia para reducir la discriminación, incrementar la justicia social, la inclusión y la calificación en las cárceles en esta era digital. En este estudio se presentan prácticas, experiencias, programas y proyectos de Educación Digital en las cárceles de todo el mundo, destacando el caso del trabajo desarrollado en Portugal por la Universidade Aberta (UAb) en colaboración con la Dirección General de Reinserción y Servicios Penitenciarios (DGRSP), sobre la creación y desarrollo del Campus Virtual Educonline @ pris, que se asume como una academia de educación, formación, empleabilidad y ciudadanía digital. Pedagógicamente inspirado en el modelo de aprendizaje de la UAb, este Campus busca dar respuesta a las necesidades educativas específicas de los estudiantes en situaciones de privación de libertad, respetando las limitaciones legales inherentes a su condición.

Palabras-clave: Educación digital. Prisiones. Inclusión. Campus virtual.

\section{Introdução}

Num mundo cada vez mais globalizado, numa sociedade de conhecimento e em rede, imersa numa realidade cada vez mais digital, existe o "risco de criar um fosso cada vez maior entre os que têm acesso aos novos conhecimentos e os que deles são excluídos" (ESTRATÉGIA DE LISBOA, 2000).

O acesso ao conhecimento, nomeadamente através da educação e da formação, como agente de inclusão social está na lista de prioridades de diversos organismos internacionais e a preocupação com grupos minoritários, como a população prisional é evidente. Como se sabe, a reclusão implica a perda de alguns direitos, mas estes não se devem estender à educação, na medida do possível, sobretudo, porque a educação e a formação, em contexto prisional, tendem a assumir-se como um dispositivo promotor de reinserção social e de combate à reincidência.

Efetivamente, hoje, assume-se a educação como um direito universal que desempenha um papel crucial no desenvolvimento humano, ajudando o indivíduo a construir a sua personalidade e o seu carácter. E mesmo em situação de reclusão, de acordo com as Recomendação N. ${ }^{\circ} \mathrm{R}(89) 12$ do Conselho da Europa ${ }^{2}$ referentes à educação em contexto prisional, os cidadãos/ reclusos possuem os mesmos direitos no acesso à educação.

2 Recomendação N. ${ }^{\circ}$ R(89)12 sobre Educação nas Prisões, adotada pelo Comité de Ministros dos Estados Membros do Conselho da Europa em 13 de Outubro de 1989 (429a Reunião de Delegados dos Ministros). 
Na realidade, os adultos em situação de reclusão mantêm o direito de acesso à educação, de qualidade e diversificada (Council of Europe Recommendation on Education in Prison, 2011), sendo que esta deve ser adequada às exigências da "Sociedade de Informação". A adoção de modelos de formação em Educação a Distância (EaD) e eLearning que possibilitem o desenvolvimento de competências-chave de literacia, assim como a relação entre a formação que é dada no interior das prisões e no sistema educacional do país, assumem-se como fundamentais para alcançar esta educação de qualidade.

Com efeito, a modalidade de Educação a Distância e eLearning tem sido reconhecida, desde o início do século, como uma modalidade que responde aos desafios que o mundo globalizado coloca ao nível da aprendizagem ao longo da vida e do desenvolvimento de competências tecnológicas e sociais (GARRISON; ANDERSON, 2003; HERRINGTON; REEVES, OLIVER, 2010; MOORE ET AL, 2011). Considerando, a importância crescente que as Tecnologias de Comunicação Digitais (TCD) têm assumido na sociedade, os reclusos devem ter a oportunidade de utilizar Ambientes Virtuais de Aprendizagem (AVA), com propósitos formativos e educacionais, obviamente, com restrições em termos de acesso à internet através de canais seguros (ADAMS; PIKE, 2012).

No entanto, e apesar da relevância assumida pela Educação a Distância e eLearning, na criação de ambientes de aprendizagem inclusivos e no desenvolvimento de competências digitais, imprescindíveis para a inclusão digital e social, os estabelecimentos prisionais, dos diferentes países europeus, de uma forma geral, têm oferecido acesso muito limitado às TDC e aos AVA, sobretudo, justificado por questões de segurança, como referido por Munoz (2009) e Hawley, Murphy e Souto-Otero, (2013), ou por falta de recursos, financeiros, tecnológicos ou digitais como destacado pelo estudo realizado em 2013 pelo Observatório Europeu das Prisões, em que participaram a Letónia, a Polónia, a Espanha, o Reino Unido, Portugal, a Itália, a Grécia e a França (MACULAN; RONCO; VIANELLO, 2013) e sublinhado, mais recentemente, por Manjer, Eikeland e Asbjørnsen (2019) e Renbarger, Rivera e Sulak (2019).

Esta realidade evidencia a atualidade e pertinência deste artigo e justifica a necessidade de produzir conhecimento acerca dos desafios educacionais e das potencialidades da Educação Digital enquanto estratégia para reduzir a discriminação, aumentar a justiça social, a inclusão e a qualificação em prisões. Neste texto são apresentadas algumas práticas, experiências, programas e projetos de Educação Digital em prisões por todo o mundo, com especial destaque para o trabalho desenvolvido em Portugal pela Universidade Aberta (UAb) em parceria com a Direção Geral de Reinserção e Serviços Prisionais (DGRSP), relativo à criação e desenvolvimento do 
Campus Virtual Educonline@pris, que se assume como uma academia de educação, formação, empregabilidade e cidadania digital.

\section{Programas de integração do digital em prisões}

Mas apesar destas limitações e dificuldades, e assumindo a necessidade de investir neste domínio têm surgido, desde o início do Século XX, sobretudo, na Europa, projetos financiados por programas Europeus de Aprendizagem ao Longo da Vida, enquadrados em subprogramas, tais como o Grundtvig e o Leonardo da Vinci, que procuram explorar o potencial das TDC e dos AVA em contexto prisional, criando soluções personalizadas em função das questões de segurança, de políticas e/ou legais, entre outras.

Em termos gerais, os resultados destes programas associados ao uso das TDC e de AVA, revelou que é possível desenvolver sistemas de segurança e de gestão de aprendizagem adaptados às distintas necessidades organizacionais e pedagógicas das diferentes prisões (MONTEIRO; MOREIRA; LEITE, 2016).

Ainda no que diz respeito a projetos europeus em contextos prisionais, é de realçar que alguns países, após a participação nesses programas, com duração limitada, encontraram as suas próprias soluções, como por exemplo o projeto Elis, desenvolvido pela Alemanha e pela Áustria, o projeto "Internet for Inmates", desenvolvido pela Noruega e o projeto "Prison Education Basic skills Blended Learning (Pebble)”, desenvolvido em parceria pela Itália, Roménia, Grécia e Chipre.

Na Alemanha e na Áustria, a plataforma Elis foi desenvolvida em 2004 tendo por objetivo garantir acesso imediato a conteúdos educacionais em contexto prisional. Sob o ponto de vista técnico, foi utilizada uma plataforma open source -ILIAS-, adaptada para ir ao encontro dos requisitos do eLearning em prisões, sendo que o acesso à rede era restrito a uma lista de endereços autorizados, uma whitelist.

Na Noruega, a rede IFI - "Internet for Inmates” serve a totalidade das prisões. Este sistema utiliza recursos oriundos da Internet, organizados em categorias, sendo que as pessoas reclusas apenas têm acesso às categorias consideradas seguras (HAMMERSCHICK, 2010).

Por sua vez o projeto Pebble, que tinha como principal objetivo ajudar os reclusos a adquirir competências chave de literacia, numeracia, competência digital e habilidades financeiras, envolveu o desenvolvimento de um sistema de blended learning, com integração de ambientes virtuais e físicos de aprendizagem. 
De destacar, ainda, em Portugal, o projeto eLearning em estabelecimentos prisionais, (E-PRIS), concebido e desenvolvido pelo Instituto Piaget, em parceria com a Direção Geral de Reinserção e Serviços Prisionais e a Santa Casa de Misericórdia do Porto, que teve como principal objetivo a criação de "modelos de intervenção integrada e estruturada, suscetível de replicação/disseminação, com o recurso às tecnologias de informação e comunicação (TIC) enquanto instrumento de inclusão digital e social" (MONTEIRO et al., 2018, p. 132). Partindo das potencialidades do eLearning como dispositivo de diferenciação pedagógica promotor de inclusão digital, a conceção do projeto permitiu que o E-PRIS articulasse as questões da formação e aprendizagem ao longo da vida com a investigação e produção científica, associada ao desenvolvimento de novos modelos pedagógicos.

De referir, ainda, outros programas mais recentes que têm promovido o uso das tecnologias digitais como forma de reabilitar os reclusos. Entre estes, destaque para o programa PrisonCloud, criado em 2016 na prisão de Beveren, na Bélgica, que permite o acesso, com restrições, a serviços online nas celas dos reclusos. O PrisonCloud assume-se como um serviço digital seguro que permite aos reclusos aceder à TV, filmes de vídeo, a programas de eLearning e a páginas da web, nomeadamente sobre cuidados de saúde, pesquisas de emprego e questões legais. As autoridades prisionais da Bélgica têm sido alvo de várias críticas, mas, no entanto, não deixam de defender a ideia de que "mudanças na sociedade, exigem mudanças na prisão."

Por sua vez, em Agder, na Noruega, iniciou-se em 2020 um projeto que tem como principal aumentar a qualidade do tratamento prisional, por via da inclusão da tecnologia. Nesta “prisão digital”, tal como é definida pelos responsáveis do programa, o principal desafio reside no trabalho conjunto desenvolvido pelos diferentes atores prisionais: escola, saúde, agências de trabalho, oficinas, bibliotecas etc. e na capacidade de assumir o digital como elemento catalisador de todo um processo de empowerment da população reclusa, mormente através de uma aposta efetiva em áreas como a educação e a reabilitação.

Também os Serviços Prisionais de Hong Kong se encontram a desenvolver um projeto intitulado Smart Prison com o intuito de adequar o tratamento prisional à atual dinâmica de desenvolvimento das sociedades. As autoridades prisionais da antiga colónia inglesa, encontram-se a analisar a possibilidade de providenciar aos reclusos um dispositivo digital (tablet) que lhes permita comunicar com familiares e amigos no exterior da prisão, bem como com advogados e funcionários durante as horas de lazer (o conteúdo será supervisionado antes de ser enviado), nomeadamente através de correio eletrónico, sendo que tal recurso seria igualmente utilizado 
em programas de eLearning, garantindo o acesso a eBooks e promovendo condições favoráveis de estudo ${ }^{3}$.

Em alguns Estados dos EUA, por seu turno, as autoridades prisionais permitem que os reclusos tenham acesso a tablets, com livros e vídeos educativos. O envio de e-mails (segundo uma lista pré-aprovada de contactos) e a possibilidade de fazerem chamadas telefónicas, adquirir músicas e videojogos, de apresentar queixas, aceder a bibliotecas ou de participar em cursos de capacitação profissional, são algumas das valências a que estes reclusos também têm acesso ${ }^{4}$.

\section{Projetos de educação digital em rede para a educação superior}

Para além dos projetos e programas atrás enunciados existem outros de maior dimensão, quer na Europa, ou fora dela, associados à frequência de cursos de Ensino Superior que envolvem, na sua maioria, as instituições universitárias de Educação a Distância nacionais. São os casos do Virtual Campus do Reino Unido desenvolvido pela Open University, o Programa de Estudios Universitarios en Centros Penitenciarios (PECP) da Universidad Nacional de Educación a Distancia (UNED) de Espanha, o programa Making the Connection da University of Southern Queensland na Austrália e o projeto da National Open University da Nigéria.

Relativamente ao Virtual Campus do Reino Unido (Inglaterra e País de Gales), do ponto de vista tecnológico, a plataforma foi desenvolvida pela Open University, em articulação com os parceiros do setor da justiça, com o objetivo de gerir as diferentes necessidades das pessoas reclusas, que têm acesso seguro a recursos digitais e conteúdos específicos, numa "whitelist" previamente definida (OPEN UNIVERSITY, 2019). Foi implementado na maioria das prisões e tem como objetivo agilizar e modernizar o sistema educativo a nível do Ensino Superior (TURLEY; WEBSTER, 2010). O Campus apresenta algumas limitações de navegação, porque na realidade não foi projetado para simular uma experiência de navegação livre na Internet, mas sim para uma navegação em ambiente controlado e seguro numa plataforma onde os estudantes/reclusos acedem aos e-conteúdos. A Open University disponibiliza também um serviço de aconselhamento profissional que presta apoio ao estudante/recluso, quer em situações de transferência de estabelecimento ou em situações de fim de cumprimento de pena. No que diz respeito a apoios financeiros, é de realçar que a frequência universitária de estudantes/reclusos já foi apoiada por fundos públicos, no entanto, o governo, optou, recentemente, por não fazer

3 Em South China Morning Post, 17 de junho, 2019 (edição online) <https://www.scmp.com/news/hong-kong/law-and-crime/article/3014714/hongkong-prison-service-studying-scheme-give-all>.

4 Em Reuters, 18 de junho, 2018 (edição online) <https://www.reuters.com/article/us-usa-prisons-computers/in-u-s-prisons-tablets-open-window-tothe-outside-world-idUSKBN1K813D>. 
uma discriminação positiva, não privilegiando o cidadão recluso perante o cidadão em liberdade. Refira-se, no entanto que o estudante/recluso com dificuldades económicas e sem capacidade de recorrer a empréstimos pode socorrer-se de organizações não-governamentais, como a Prisoners' Education Trust (PET) (COATES, 2016).

Por sua vez, em Espanha o Ensino Superior nas prisões é assegurado em exclusivo pela Universidad Nacional de Educación a Distancia (UNED), através do Programa de Estudios Universitarios en Centros Penitenciarios (PECP), cujo objetivo é aumentar o nível de formação da população reclusa, possibilitando, através da Educação a Distância, a frequência do Ensino Superior (UNED, 2019). Este programa está regulado por acordos assinados entre o Ministério da Educação, Cultura e Desporto, o Ministério da Defesa, a Secretária Geral das Instituições Penitenciárias (SGIP) do Ministério do Interior e a UNED.

Às prisões compete estabelecer a forma em como o PECP será desenvolvido no contexto prisional (espaços de estudo, tempo e meios disponibilizados) e assegurar a informação geral e a gestão administrativa do PECP através dos seus Gestores da Formação e Inserção Profissional (GFIP).

A metodologia da UNED permite que os estudantes realizem de forma autónoma grande parte do trabalho necessário à aprovação das unidades curriculares dos cursos, através do material didático disponibilizado em suporte de papel (textos básicos e materiais de apoio). Para além disso os estudantes/reclusos têm acesso, em várias prisões, à Plataforma aLF (Aprende, coLabora, Forma) da UNED - "plataforma virtual educativa para el aprendizaje y el trabajo colaborativo en- línea, que permite ofrecer y recibir formación através de cursos y comunidades virtuales" (UNED, 2019, p. 16), que permite o acesso a material multimédia - acessível com o apoio de assistentes da UNED.

Os estudantes/reclusos podem contactar com os docentes através de contacto telefónico (de acordo com o regime de cada prisão) e/ou correio postal. A UNED organiza as provas de avaliação presencial que são realizadas nas prisões designadas como prioritárias. Os estudantes/ reclusos que se encontrem noutras prisões podem solicitar antecipadamente a deslocação para a realização das provas. A UNED assegura ainda a existência do seu material pedagógico nas bibliotecas das prisões.

De forma a contornar as disparidades e dificuldades económicas dos estudantes/reclusos a frequência do PECP é gratuita (sempre e quando cumpram as condições do programa).

O processo de implementação do PECP e do acesso à Plataforma aLF é desenvolvido conjuntamente pelos técnicos da UNED e das prisões e assenta nos seguintes pressupostos:

a) A navegação e as comunicações são controladas constantemente, de modo a que se 
possa controlar e supervisionar todo o tráfego de informação entre os estabelecimentos prisionais e a UNED;

b) É assegurado que os estudantes/reclusos, através desta ligação, só conseguem aceder aos espaços educativos digitais da UNED/Plataforma digital de aprendizagem;

c) O acesso dos estudantes à plataforma digital é condicionado. Têm acesso aos conteúdos programáticos, à avaliação contínua, às provas e aos exemplos apresentados pelo docente. Não lhes é permitido o acesso às ferramentas comunicacionais da plataforma (fóruns, mensagens privadas salas de chat, videoconferências online ou outros) ou a ligações externas.

d) Os estudantes/reclusos não conseguem submeter trabalhos através da plataforma, pois é considerada uma forma de comunicação. A entrega deve ser feita ao assistente da UNED que está presencialmente no estabelecimento prisional ou através dos meios tradicionais (VIEDMA-ROJAS, 2017).

No caso da Austrália é de destacar que os reclusos têm acesso ao Ensino Superior, sobretudo, através de um projeto, financiado pelo governo australiano, intitulado Making the Connection, que se encontra a ser desenvolvido com o apoio da University of Southern Queensland (USQ).

Para concretizar este projeto de educação digital foi instalada uma versão da plataforma digital da USQ nos servidores das prisões participantes e distribuídos computadores portáteis já com recursos e conteúdos dos cursos de graduação frequentados pelos estudantes/reclusos. Estes conteúdos e recursos foram adaptados para consulta sem acesso à Internet, numa versão USQ Offline StudyDesk com computadores portáteis.

Para além deste projeto, é de destacar ainda na Austrália, o PLEIADES (Portable Learning Environments for Incarcerated Distance Education Students), um projeto concebido para fornecer aos estudantes reclusos acesso a tecnologias móveis e digitais seguras offline e que tinha como principal objetivo aumentar o acesso e a participação em cursos de Ensino Superior. Para esse efeito foi desenvolvida uma versão offline da Moodle para complementar a leitura dos recursos digitais disponibilizados em eReaders (FARLEY; MURPHY; BEDFORD, 2014).

Na Nigéria os únicos reclusos que têm acesso a computadores são os estudantes/reclusos que frequentam cursos de Ensino Superior na Universidade Nacional Aberta, no entanto, estes, também, não têm autorização para aceder à Internet. A única exceção acontece durante a ápoca de realização de exames, já que nesse período a Universidade Nacional Aberta da Nigéria cria um acesso à rede digital através de uma Rede de Área Local (LAN) e de um servidor proxy da universidade nas salas de exame, criadas para os estudantes/reclusos, localizadas no interior dos pátios das prisões. Após a conclusão dos exames, que duram quase oito semanas, a rede e o servidor proxy são retirados desses espaços. 
Os recursos educativos são fornecidos pela universidade em versão impressa e digital, sendo que muitos desses recursos estão disponíveis nos computadores e outros são entregues em CD-Rom. Os estudantes/reclusos, normalmente, realizam os seus trabalhos em formato manuscrito e, depois disso, os Conselheiros de Estudantes Universitários recolhem esses trabalhos e realizam o upload em nome dos estudantes para o portal da universidade Computer Marked Assignment (CMA) onde são avaliados pelos professores. Depois dessa avaliação os resultados são impressos e devolvidos aos respetivos estudantes.

\section{O Campus virtual de educação, formação, cidadania e empregabili- dade: Educonline@Pris}

Também em Portugal, e no âmbito do protocolo assinado em abril de 2016 entre a Universidade Aberta (UAb) e a Direção Geral de Reinserção e Serviços Prisionais (DGRSP) onde se destacava a necessidade e o compromisso de criar e desenvolver um Campus Virtual, concebido para a população reclusa, com acesso seguro, para o desenvolvimento de atividades no domínio do ensino e formação em Educação a Distância e eLearning (Cláusula 2. ' - Cooperação), está a ser desenvolvido um projeto-piloto inovador, o Campus Digital Educonline@Pris,

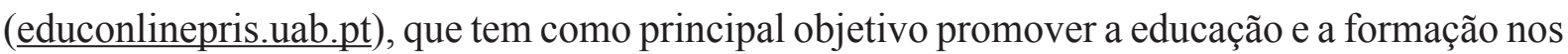
estabelecimentos prisionais em Portugal em ambientes virtuais de aprendizagem.

E foi na sequência desse protocolo e do definido nessa Cláusula 2. ${ }^{a}$ que se começou a desenhar e a desenvolver um projeto disruptivo que se está a materializar num Campus Virtual -Educonline@Pris- (educonlinepris.uab.pt).

Depois de um primeiro ano de implementação, numa versão piloto, em quatro estabelecimentos prisionais do Norte do país, com resultados promissores face à utilização do Campus Virtual (SILVA; MOREIRA; ALCOFORADO, 2019), a rede foi alargada no final do ano de 2020, para os cerca de vinte estabelecimentos prisionais com reclusos-estudantes a frequentar cursos de Licenciatura e Mestrado da Universidade Aberta, tendo sido alocados a estes estabelecimentos prisionais Laboratórios de Aprendizagem Móveis, com computadores portáteis e conectividade.

O Campus Virtual Educonline@pris possui um portal agregador, tendo por base quatro plataformas, uma que dá acesso aos cursos de licenciatura, mestrado e doutoramento da Universidade Aberta -eLearning UAb-; outra com cursos e ações de extensão universitária,-ON@Pris-, desenvolvida, especificamente, para a população reclusa, com formações certificadas com o selo da iniciativa governamental INCoDE (https://www.incode2030.gov.pt), uma terceira plataforma -Aula Aberta-, que permite ao estudante recluso aceder a recursos e conteúdos de diferentes áreas 
disciplinares disponíveis na Universidade Aberta; e uma quarta plataforma -Portal Académico UAb- que integra um conjunto de serviços, permitindo a centralização da gestão de utilizadores e respetivos perfis, gestão das inscrições e de eventos ou alertas específicos.

Tal como o Campus da Open University e a Plataforma aLF da UNED, o Campus Virtual Educonline@Pris apresenta algumas especificidades e limitações de navegação, porque na realidade, também, não foi projetado para simular uma experiência de navegação livre na Internet, mas sim para uma navegação em ambiente controlado e seguro. No entanto, e apesar da navegação condicionada, a flexibilidade e a inclusão do Modelo Pedagógico Virtual ${ }^{\circledR}$ da Universidade Aberta (Pereira, et al., 2007; Mendes et al., 2018), dois dos seus princípios fundamentais, permite que estes estudantes, com um perfil de utilizador diferenciado, sejam integrados nas turmas regulares e não em "guetos" digitais.

Com efeito, o Modelo Pedagógico Virtual ${ }^{\circledR}$ da Universidade Aberta permite que os estudantes/reclusos realizem de forma autónoma grande parte do trabalho necessário à aprovação das unidades curriculares dos cursos em que estão inscritos, através dos recursos disponibilizados na plataforma digital, já que a interação é, exclusivamente, centrada nos conteúdos, sendo que não existe interação dos estudantes/reclusos com a restante comunidade virtual, nem com os professores, nem com a turma onde está inserido. O perfil do estudante/recluso apenas lhe permite aceder aos espaços de comunicação e de interação da plataforma, mas não permite que comunique ou interaja com os outros atores humanos da comunidade virtual. Apesar desta limitação ao estudante/ recluso é permitido o acesso aos conteúdos programáticos e recursos "fechados" na plataforma e às diferentes modalidades de avaliação: contínua e final, sendo que podem submeter as provas eletrónicas (e-fólios) na plataforma digital e realizar as provas presenciais (p-fólios e exames) no estabelecimento prisional com a supervisão de técnicos superiores designados para o efeito.

O sistema de acessibilidade ao Campus Virtual Educonline@Pris foi desenvolvido conjuntamente pelos Serviços Informáticos da Universidade Aberta e da Direção Geral de Reinserção e Serviços Prisionais, sendo que, como no caso da UNED, a navegação e as comunicações são controladas constantemente, de modo a que se possa controlar e supervisionar todo o tráfego de informação. Através desta monitorização conjunta entre as duas instituições e a empresa de telecomunicações associada, é assegurado que os estudantes/reclusos só conseguem aceder aos espaços educativos do Campus Virtual Educonline@Pris.

Aquando da sua criação a expectativa era que o Campus desse uma resposta efetiva às necessidades dos estudantes da Universidade Aberta em situação de reclusão, incrementando significativamente a qualidade da educação digital nos estabelecimentos prisionais (até então 
quase inexistente em Portugal). Em simultâneo, o Campus Virtual seria também uma forma de responder aos desafios colocados pela nova sociedade digital e em rede, contribuindo para assegurar o direito de todos os cidadãos à educação. E parece que esses objetivos estão a ser alcançados, tendo em conta a leitura e análise dos resultados da implementação do projeto piloto no ano de 2019 (SILVA; MOREIRA; ALCOFORADO, 2019).

\section{Considerações finais}

A experiência partilhada através dos resultados dos projetos apresentados e de estudos acerca do eLearning em prisões, permitem perceber que a Educação a Distância e o eLearning têm, ainda, um potencial imenso por explorar neste contexto. Lockitt (2011) referindo-se a este potencial destaca, sobretudo, os aspetos relacionados com a Individualidade, por exemplo, a nível de estilos de aprendizagem individuais; das metas e objetivos individuais; da aprendizagem independente; e da possibilidade de diferenciação pedagógica; com a Flexibilidade, em termos de conteúdos de aprendizagem; de recursos multimédia de aprendizagem; da interatividade; da autoavaliação; e de tecnologias móveis; e com a Continuidade, a nível de um curriculum online (registo do historial de formação e das aprendizagens desenvolvidas pelos sujeitos); da continuidade da formação prisão/comunidade; e de um apoio "virtual" consistente.

Para além das potencialidades, no mesmo estudo Lockitt identificou uma série de limitações relativamente ao recurso à Educação a Distância e ao eLearning nestes espaços de reclusão que é necessário ultrapassar, como por exemplo, acesso muito limitado à tecnologia e sinal digital e uso não efetivo dessa tecnologia; falta de sensibilização dos profissionais dos estabelecimentos prisionais acerca do potencial das tecnologias; falta de materiais multimédia interativos de qualidade; falta de apoio interativo contínuo, através de videoconferências; publicidade adversa e má perceção da sociedade quanto ao uso da tecnologia (2011).

Todas estas limitações reforçam a necessidade da implementação da Educação Digital em prisões, com um plano estratégico bem definido que considere as diversas variáveis referidas por Attwell (2006), nomeadamente, as variáveis individuais dos reclusos (características pessoais, sociais e culturais, a história de aprendizagem/ experiência anterior, atitudes, motivações, a familiaridade com tecnologias, compromisso pessoal de aprendizagem,...); as variáveis institucionais (ambiente de aprendizagem, contexto econômico e político, eLearning confiança/ valor); as variáveis tecnológicas (hardware, software, acesso, segurança e soluções de suporte técnico); bem como as variáveis pedagógicas (acessibilidade, recursos educacionais, métodos 
pedagógicos, autonomia, interatividade, flexibilidade, organização curricular, monitoramento e apoio tutorial).

Consideradas todas estas variáveis, modalidade de Educação a Distância, pode, efetivamente, contribuir para a promoção da inclusão (social e digital), através do desenvolvimento de uma educação digital em rede, entendida como o conhecimento, a atitude, a capacidade do indivíduo de usar corretamente as tecnologias digitais e as redes, bem como a capacidade para identificar, ter acesso, integrar, avaliar, analisar, resumir, criar e comunicar usando recursos digitais.

Regressando aos pressupostos iniciais deste artigo, parece-nos evidente o potencial da introdução da Educação Digital em contextos de reclusão. Acreditamos que este projeto consiga dar resposta a alguns dos desafios que a sociedade digital e as tecnologias de comunicação digital colocam à Educação Digital especialmente em contextos de enorme vulnerabilidade social, como é o caso do contexto prisional, contribuindo, ao mesmo tempo, para que seja garantido o direito de acesso à educação que deve ter qualquer cidadão, no cumprimento do respeito pelos direitos humanos dos indivíduos, privados ou não de liberdade.

A literatura também tem sido clara na demonstração de como a educação é fundamental para responder às necessidades daqueles que se encontram no limiar da exclusão social, em risco e vulnerabilidade. Este será um caminho para aumentar a justiça social e reduzir a discriminação através da frequência de cursos de educação e formação.

\section{Referências}

ADAMS, A.; PIKE, A. Digital exclusion or learning exclusion? Amn ethnographic study of adult male distance learners in English prisons. Research in Learning Technology, v. 20, n. ${ }^{\circ} 4$, p. 363-376, 2012.

ATTWELL, G. Personal Learning Environments-the future of eLearning? eLearning Papers, v.2, n. ${ }^{\circ}$ 1, 2007, Disponível em: www.elearningpapers.eu

COATES, D. S. Unlocking Potential A review of education in prison. UK: Ministry of Justice, 2016. Disponível em: https://assets.publishing.service.gov.uk/government/uploads/ system/uploads/attachment_data/file/524013/education-review-report.pdf

COUNCIL OF EUROPE. Recommendation of the Committee of Ministers to member states on the European Prison Rules, 2011. Disponível em: https://wcd.coe.int/ViewDoc. jsp?id=955747.

ESTRATÉGIA DE LISBOA. Conselho Europeu de Lisboa, 23 e 24 de Março de 2000, Conclusões da Presidência, 2000. 
FARLEY, H.; MURPHY, A.; BEDFORD, T. Providing simulated online and mobile learning experiences in a prison education setting: Lessons learned from the PLEIADES pilot project. International Journal of Mobile and Blended Learning, v. 6, n. ${ }^{\circ}$ 1, p. 17-32, 2014.

GARRISON, D. R.; ANDERSON, T. E-learning in the 21stcentury. London: RoutledgeFalmer, 2003.

HAMMERSCHICK, W. Report on e-learning in European prisons - Concepts, organisation, pedagogical approaches in prison education, 2010. Disponível em: http://www. adam-europe.eu/prj/3840/prj/08-lc-report-e-learning-in-prison-eu10.pdf

HAWLEY, J.; MURPHY, I.; SOUTO-OTERO, M. Prison Education and Training in Europe Current State-of-Play and Challenges. European Commission: GHK Consulting, 2013. Disponível em: http://www.antoniocasella.eu/nume/Hawley_UE_education_may13. pdf

HERRINGTON, J.; REEVES, T.; OLIVER, R. A guide to authentic e-learning. New York: Routledge, 2010.

LOCKITT, W. Technology in prisons, 2011. Disponível em: http://www.wcmt.org.uk/sites/ default/files/migrated-reports/797_1.pdf

MACUlAN, A.; RONCO, D., VIANELLO, F. Prison in Europe: overview and trends. Roma: European Prison Observatory, 2013. Disponível em: http://www.prisonobservatory. org/upload/PrisoninEuropeOverviewandtrends.pdf

MANGER, T.; EIKELAND, O.J.; ASBJØRNSEN, A. Why do not more prisoners participate in adult education? An analysis of barriers to education in Norwegian prisons. International Review of Education, n. ${ }^{\circ}$ 65, p. 711-733, 2019.

MENDES, A.; BASTOS, G.; AMANTE, L.; AIRES, L.; CARDOSO, T. Modelo Pedagógico Virtual. Cenários de desenvolvimento. Lisboa: Universidade Aberta, 2018.

MONTEIRO, A.; MOREIRA, J. A.; LEITE, C. O eLearning em estabelecimentos prisionais: possibilidades e limites para a inclusão digital e justiça social. Revista Diálogo Educacional, v. 16, n. ${ }^{\circ}$ 47, p. 77-102, 2016. Disponível em: http://www2.pucpr.br/reol/pb/index.php/dialog o?dd $1=16098 \& d d 99=$ view $\&$ dd $98=\mathrm{pb}$

MONTEIRO, A.; LEITE, C.; BARROS, R. Eu ganhei mais o gosto de estudar: O e-learning como um meio de Aprendizagem ao longo da vida de reclusas de um Estabelecimento prisional português. Educação e Sociedade, v. 39, n. ${ }^{\circ}$ 142, p. 129-150, 2018. Disponível em: http://www.scielo.br/pdf/es/v39n142/1678-4626-es-ES0101_7330216156650.pdf

MOORE, J.; DICKSON-DEANE, C.; GALYEN, K. e-Learning, online learning, and distance learning environments: Are they the same? The Internet and Higher Education, v. 14 n. ${ }^{\circ} 2$, p. 129-135, 2011.

MUÑOZ, V. EI derecho a la educación de las personas privadas de libertad. Asamblea General Consejo de Derechos Humanos - ONU, 2009. Disponível em: https://cdn.penalreform.org/wp-content/uploads/2013/06/UNHRC_Ed2009_sp_0.pdf 
PEREIRA, A.; MENDES, A. Q.; MORGADO, L.; AMANTE, L.; BIDARRA, J. Modelo Pedagógico Virtual da Universidade Aberta. Lisboa: Universidade Aberta, 2007.

RENBARGER, R.; RIVERA, G.; SULAK, T. What predicts literacy, numeracy, and problem solving for incarcerated individuals? A PIAAC examination. Journal of Offender Rehabilitation, v. 58, n. ${ }^{\circ} 3$, p. 199- 219, 2019.

SILVA, S.; MOREIRA, J. A.; ALCOFORADO, L. Educação Digital no Ensino Superior em Portugal em Contexto de Reclusão: o Campus Virtual Educonline@Pris. EccoS - Revista Científica, v. 51, n. 4, p. 1-22, 2019. Disponível em: https://periodicos.uninove.br/index.php ?journal $=$ eccos\&page $=$ article $\&$ op $=$ view $\&$ path $\% 5 \mathrm{~B} \% 5 \mathrm{D}=16256$

THE OPEN UNIVERSITY. Make a New Start Studying with The Open University - A Guide For Learners in Secure Environments 2019/2020. UK: The Open University, 2019. Disponível em: http://www.open.ac.uk/secure-environments/sites/www.open.ac.uk.secureenvironments/files/files/Prospectus\%20_SiSE1819.pdf

TURLEY, C.; WEBSTER, S. Implementation and Delivery of the Test Beds Virtual Campus Case Study, National Centre for Social Research, 2010. Disponível em: https://www. gov.uk/government/uploads/system/uploads/attachment_data/file/31466/11-827-implementation-of-test-beds-virtual-campus.pdf.

UNED. Guía práctica: Conocer y utilizar el Campus UNED y los Cursos Virtuales en aLF Docente. Madrid: UNED, 2019. Disponível em: https://descargas.uned.es/publico/pdf/ Guiapractica_aLF.pdf

VIEDMA ROJAS, A. La situación de la educación en las cárceles de España: realidades y expectativas de transformación. In: Los derechos de los reclusos y la realidad de las cárceles españolas. Perspectivas sociales, políticas, jurídicas y filosóficas. p. 95-117, 2017. Disponível em: https://www.researchgate.net/publication/318852947_La_situacion_de_la_educacion_ en_las_carceles_de_Espana_realidades_y_expectativas_de_transformacion

Recebido em: 20 de fevereiro de 2021.

Publicado em: 20 de abril de 2021. 